\title{
Using Blog Comments as Feedback to Promote the Metacognitive Development of Creativity
}

\author{
McMahon, Mark'; Heather, Joseph ${ }^{\mathrm{a}}$
}

${ }^{a}$ School of Arts and Humanities, Edith Cowan University, Australia.

\begin{abstract}
Creativity can be viewed, not just as a set of skills and strategies, but as an overarching metacognitive skill that integrates a range of subordinate generic skills. Key to developing creativity is to engage in a cycle of ideation, reflection and adjustment, within a feedback rich environment. Blogs have the ability to garner external comments that can prompt these processes. Case study research was undertaken to explore what forms of feedback promote metacognitive development and how those forms can best be elicited within a blog. Findings indicated that blog comments can motivate, provide information, enhance quality and promote reflection, and that a range of strategies can be applied in blogs to best obtain the most valuable forms of feedback for creative development.
\end{abstract}

Keywords: weblogs; blogs; feedback; metacognition; creativity, reflection. 


\section{Introduction}

One approach to teaching creativity is to teach for creativity rather than simply modeling creative strategies (Kaufman \& Sternberg, 2007). As a generic skill, it can be addressed through a range of related skills and attributes, such as critical thinking and information literacy. Clements and Nastasi (1999) foreground the role not of knowledge itself but knowledge acquisition strategies. Such strategies 'relate newly acquired information to information acquired in the past. Knowledge-acquisition components are fundamental sources of learning, insight, and creativity.' (Clements \& Nastasi, 1999, p. 12)

Associated skills that have been identified include information management, selforganisation, and ability to manage risk as well as reflection, self-evaluation, and interpersonal skills (Gourley, 2003). This positions creativity as a metacognitive skill that is applied and developed through a high level of self-awareness within the practitioner.

This paper outlines a case study undertaken by an honours research student using a blog as a tool for personal creative development within the context of game concept art. The blog provided the basis for publishing his developing art over a 6 month period. The aim was to explore how a metacognitive view of creativity could inform creative development, with a particular focus on the value of feedback garnered through blogging in supporting the iterative processes of ideation, reflection and adjustment required to evolve creative ideas.

\section{A Metacognitive Approach to Developing Creativity}

Perfect and Schwartz (2002) synthesise much of the literature on metacognition as a concentration on the interactive mechanisms of monitoring and control, in relation to achieving a certain goal. Monitoring refers to an individual's ability to judge their cognitive processes, and control refers to their ability to adjust and strategize from this knowledge. This identifies the relationship between creative thought (creativity-relevant skills) and the creative act (domain-relevant skills). Metacognition in this sense is comprised of three components: (a) awareness of a problem; (b) planning and engaging the appropriate strategies to address it; and (c) monitoring and regulating the ongoing activity.

This inherently reflective approach is also manifest in models of creative agility, involving the pursuit of a particular goal (awareness), reflecting on the outcomes of ideas (monitoring), and adjusting (controlling) the initial approaches based on learnt options from that reflection (Hill, Brandeau, Truelove, \& Lineback, 2014). Creative agility does not assert that it is the one single process for creativity, rather that is mediates a range of subordinate processes such as generating ideas through debate, iterating them multiple times, integrating decisions based on goals, reflection, and adjustment, identifying problems and solutions, and so on. 
This study was informed by a model that synthesises this research (Figure 1). The key elements are both the creative and domain relevant skills as well as intentionality and feedback to mediate their development. This mediation is conducted through the metacognitive act of ideation, reflection and adjustment.

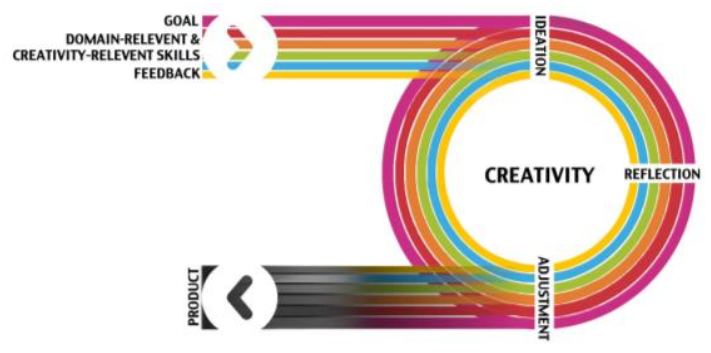

Figure 1. A metacognitive model for creative development

\section{Method}

The model was applied by an undergraduate honours student in Design through the implementation of a weblog, which acted as a visual diary, with the added affordances of communication enabled through online publishing. Posts included examples of work in progress, discussions of key themes and influences, reflections on the work as it developed and responses to feedback. Participants were recruited to provide the feedback in the form of blog comments. They included undergraduate students in Design from the same university, where critiquing the blog posts constituted a classroom activity about game concept design. Invitations were also sent via game art forums and online communities.

Feedback in the form of participant comments were then analysed to classify types of feedback with a view to identifying those that best inform creative development and provide recommendations as to how to elicit the most useful types. A constant comparative approach was used to continuously analyse comments over time, involving a variety of coding techniques

\section{Findings}

Existing research has shown that feedback can have a range of functions that provide information, boost motivation, clarify standards, facilitate reflection, and assist in skills development (Ion \& Stîngu, 2014). In combining these precepts with trends that emerged directly from the comments themselves, four roles for feedback became apparent that provided a strong basis for organizing discussion and analysis of the value of feedback in: providing information to assist skills development; strengthening motivation; prompting reflection; and facilitating good performance 


\subsection{Providing Information to Assist Skills Development}

Throughout the blog feedback, there were many instances where information was provided to assist skills development. The first key finding with regard to this was the somewhat obvious one that to receive information that supports the development of skills, an object is required that encourages participants to talk and direct their attention. Simply posting the artwork provided many positive comments but little actual information beyond congratulations and encouragement.

Better examples were when the artist posted drawings practicing the female form. These were deliberately rough in nature (Figure 2), and were accompanied by a brief discussion on human body shapes and structures that identified the drawings' need for a realistic body shape for the video game heroine. More valuable comments were then received that directed the artist to 'study how the body parts are made up of bones, sinew, musculature and fat tissue' (Participant 4) and drew attention to the anatomical drawings of Leonardo da Vinci. The external reference point of anatomical drawings enabled the recognition of weaknesses, the ability to calibrate understandings with those of others and respond by refining the work in a way that enhanced drawing skills.

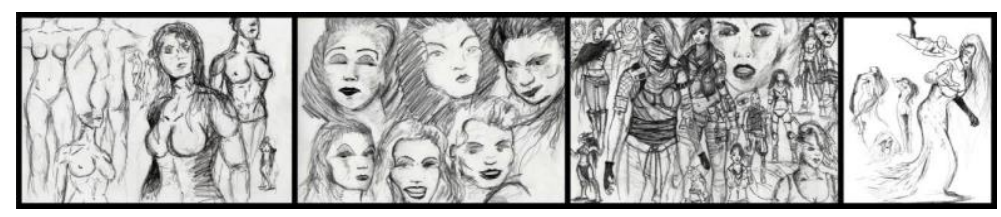

Figure 2: Rough female anatomical drawings

A second published artefact focused on integrating ideas about personality, aesthetic design and environment into a female form. The post cited multiple video game and film media. These examples enabled commentators to dissected the artefact and draw conclusions, with comments such as '...the tightness of her clothing demonstrates just how guarded she is...' (Participant 5) while another suggested art styles in Games such as Limbo and Feist for further inspiration (Participant 6). Such comments that added to the context of the drawing, furthered skills both in terms of technical (drawing) and conceptual aspects (setting, character etc.).

\subsection{Strengthening Motivation}

Motivation can be seen as a major contributing aspect of metacognitive self-regulation (e.g. Boekaerts, 1997; Garcia \& Pintrich, 1994). It moderates effort, which in turns assists in mastery of skills. One of the strongest values of feedback in this blog was its power to motivate the artist. While comments such as feedback such as 'Love this! so creative!' (Participant 1) or 'Freaky fun. Luv it' (Participant 2) do not provide information for further learning, they do provide validation and encouragement that stimulates further actions to 
reach desired ends. Published artefacts such as that shown in Figure 4 were particularly effective in prompting motivational feedback.

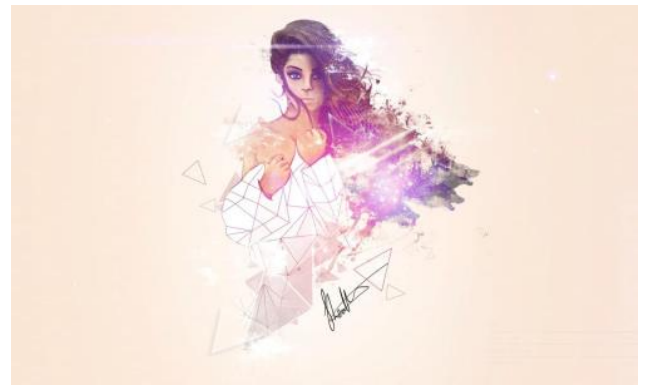

Figure 4: Evolution of female anatomical drawing

This post demonstrated how the work shown in Figure 3 was evolving and elicited comments such as, 'brilliant artwork' (Participant 7), which in turn stimulated the desire to achieve goals by boosting self-esteem (creativity-relevant skills) and self-control (domainrelevant skills). It validated the improvement of skills compared to previous work. One comment was particularly notable in that it pointed out aspects that were realistic and feminine but also pointed to specific design characteristics and illustrative techniques (Participant 8). While the comment did not provide sufficient information from which to develop skills, it did present a sense of appreciation that enhanced effort. The consideration given to what kind of works to make, how to go about doing so, and how to present them, in particular enhanced the credibility of the comment as well as prompting reflection.

A trend became apparent where artefacts that were published with a low level of refinement feedback were more likely to elicit formative than summative feedback. This may be because such works provide the community with an aesthetic quality to engage with, which further enhances the detail within the feedback, and therefore its credibility. However, although motivational feedback is valuable in sustaining one's drive to achieve a creative goal, a balance needs to be struck. Praise helped regulate the artist's effort but did not necessarily lead to specific skill development.

\subsection{Facilitating Good Performance}

One of the other problems with purely motivational feedback is that it fails to provide the means for objective judgement of performance. A few highly praiseworthy comments could skew the perception of the work, particularly with regard to its value as perceived by the broader community.

To address this, two designs were presented and the participants were asked to select which one they preferred and why (Figure 4). This provided some statistical information as well as informative comments. $63 \%$ of comments chose Design A but it was only when reviewing 
the reasoning that it became apparent why. In that sense, statistical information fails to provide knowledge about what is successful about a work but when one participant commented that the preferred image 'definitely fits the character description ... [has] way more details in it too ... [and] the smokey affect gives me more of that feeling that he's a drug addict and an alcoholic' (Participant 11), clear reasoning could be found for the general consensus provided by the statistics.
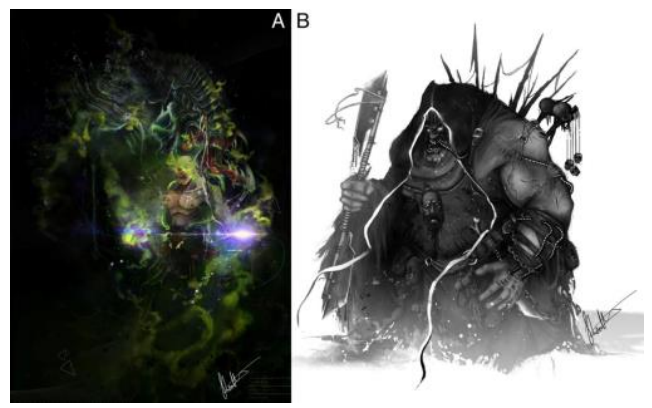

Figure 4: Two designs compared

The combination of both 'what' and 'why' were essential to good performance in that statistical summary can provide an objective measure which can then be triangulated with reasoning to facilitate good performance. In this particular case it honed the design in terms of its ability to embody the contextual elements of the game world.

\subsection{Prompting Reflection}

Reflection is perhaps the key metacognitive element for the development of creativity since it underpins the self-monitoring inherent in most of the metacognitive models. Feedback would appear to be a powerful tool as a reflective prompt but as has been shown, comments can reinforce existing biases through praise or simply provide information. This research found that certain types of comments were more valuable for prompting reflection and that specific strategies were also more successful in eliciting those types of comments.

What differentiates feedback for reflection from other forms is how it extends on the information provided to prompt individuals to consider their performance against some criteria. Using Image B of Figure 4 as an example, the artefact was published with a backstory which contextualised and broadened the character. This promoted richer feedback by providing specific features to discuss:

Participant 12 pointed out a lack of originality in the character, with its similarity to characters in Diablo 3 and World of Warcraft as well as some gaps in logic (such as the 'fat' neck and 'funny' muscles), which in turn prompted reflection on those aspects of the design that were clichéd or inaccurate. It is important to note the comment did not define exactly how to proceed with the mentioned adjustments, it did provide external reference 
points that enabled the original idea of the character to be re-addressed. As a result, the purpose and effect of every feature was reanalyzed for its coherence.

As an internal thought process, reflection is not mediated by feedback but prompted by it, with the ultimate goal of the snowballing iterative cycle of ideation and adjustment shown in Figure 1. The best feedback for reflection, then, should inspire and introduce new ideas but not lay down the path of how to necessarily execute them. This should involve connecting the current state of the creative artefact (awareness) with its intended goal (monitoring) through evaluation (controlling). From this, metacognition grows knowledge and enables one to exercise control of that knowledge. Eliciting feedback that challenges one's own assumptions, therefore, is an important strategy for prompting reflection to enhance metacognitive development of creativity.

\section{Conclusions and Recommendations}

As has been shown in this case study, feedback is a potent tool to promote the metacognitive development of creativity. Blogs are a natural medium for this as they provide both the creative flexibility of publishing ideas and artefacts while engaging broadly with a community to garner comments. That said, consideration needs to be given to the different types of feedback received. A variety of forms are required to ensure that they provide information, motivate, facilitate good performance and prompt reflection. Many social media environments allow people to post work and gain comments such as 'likes', providing a statistical and objective base to count the perceived value of a post by others. However, without comments they do not provide remediation, support, or extra information. Open ended blogging environments such as WordPress can enable richer interaction but consideration still needs to be given to what artefacts are posted and how comments are elicited to maximize the value of feedback for creative development. With that in mind, the following recommendations are made:

1. Information which assists skills development is most beneficial when feedback articulates specifics about the published artefact and provides additional information to extend learning beyond initial ideas. This is best elicited by creative works that are published with an accompanying written elaboration from which the community can draw on, to provide informative and detailed feedback.

2. Feedback which strengthens motivation is most valuable when the feedback firstly praises the artefact and secondly draws attention to elements that deemed the artefact praiseworthy. Eliciting this kind of feedback requires published artefacts with high levels of refinement.

3. The best kind of feedback for good performance is where feedback allows for some kind of objective analysis. One way is to request a level of detail in 
comments to gauge their credibility and validity. Another is to create an objective measure such as a polling system. The number of likes or votes can allow the judgment of how well a post has resonated with the audience.

4. The most valuable feedback to prompt reflection identifies the artefact's goal, draws on aspects of the artefact to provide new perspectives outside of the individual and encourages reflection on this information in terms of one's own performance. This can be elicited through publishing written elaboration with the artefact showing clear sense of goals and generate sufficient detail in the artefact to prompt links between the current state in contrast to the desired state. This then facilitates how to identify and evaluate alternatives and then select a solution.

As environments that give artists opportunities to present ideas, reflect on them, and adjust them within a feedback rich environment, blogs are an ideal medium to assist in the metacognitive development of creativity. In this socially mediated world, understanding the nature of different types of feedback and having an ability to elicit the variety of types required to motivate, inform, build quality and prompt reflection will be key skills for higher education students who seek to enhance their creativity as developing artists.

\section{References}

Boekaerts, M. (1997). Self-Regulated Learning: A new concept embraced by researchers, policy makers, educators, teachers, and students. Learning and Instruction, 7(2), 161186.

Clements, D. H., \& Nastasi, B. K. (1999). Metacognition, learning, and educational computer environments. Information Technology in Childhood Education, 4(1), 5-38.

Garcia, T., \& Pintrich, P. R. (1994). Regulating motivation and cognition in the classroom: The role of self-schemas and self-regulatory strategies. In D. H. Schunk \& B. J. Zimmerman (Eds.), Self Regulation of Learning and Performance: Issues and educational applications (pp. 127-153). Hillsdale, N. J.: Erlbaum.

Gourley, B. (2003, 4, February, 2003). We can all learn to be creative in the right environment: the challenge is to provide it. The Independent.

Hill, L. A., Brandeau, G., Truelove, E., \& Lineback, K. (2014). Collective Genius: The Art and Practice of Leading Innovation: Harvard Business Review Press.

Ion, G., \& Stîngu, M. (2014). USING FEEDBACK THROUGH BLOGS TO ENHANCE STUDENTS'SELF-REFLECTION AND LEARNING. Paper presented at the The International Scientific Conference eLearning and Software for Education.

Kaufman, J., C., \& Sternberg, R., J. (2007). Creativity. Change(39), 55-58.

Perfect, T. J., \& Schwartz, B. L. (2002). Applied Metacognition: Cambridge University Press. 